\title{
GLAD!
}

Revue sur le langage, le genre, les sexualités

$08 \mid 2020$

$G L A D$ ! en grève

\section{La peinture ne recouvre pas notre précarité}

Painting Doesn't Cover Our Precariousness

La pintura no tapa nuestra precariedad

\section{Agustina Mattaini}

\section{(2) OpenEdition}

\section{Journals}

Édition électronique

URL : https://journals.openedition.org/glad/1899

DOI : $10.4000 /$ glad. 1899

ISSN : 2551-0819

Éditeur

Association GSL

Référence électronique

Agustina Mattaini, «La peinture ne recouvre pas notre précarité », GLAD! [En ligne], 08 | 2020, mis en ligne le 01 juillet 2020, consulté le 21 septembre 2021. URL : http://journals.openedition.org/glad/ 1899 ; DOI : https://doi.org/10.4000/glad.1899

Ce document a été généré automatiquement le 21 septembre 2021.

\section{(9) $\odot \Theta \Theta$}

La revue GLAD! est mise à disposition selon les termes de la Licence Creative Commons Attribution -

Pas d'Utilisation Commerciale - Pas de Modification 4.0 International. 


\title{
La peinture ne recouvre pas notre précarité
}

\author{
Painting Doesn't Cover Our Precariousness \\ La pintura no tapa nuestra precariedad
}

Agustina Mattaini Ce média ne peut être affiché ici. Veuillez vous reporter à l'édition en ligne http://
journals.openedition.org/glad/1899

2 La peinture ne recouvre pas notre précarité apparaît avec le désir de créer une réflexion non seulement autour des grèves et des luttes qui ont eu lieu en France dès décembre 2019, mais aussi avec la volonté de se demander quelle est la place de la communauté universitaire dans ces mouvements. En prenant des photographies de manifestations parisiennes mais aussi de graffitis, collages, affiches et même de traces d'inscriptions grévistes partout en France, j'ai commencé à concevoir la grève non seulement comme une façon de (nous) rendre visibles et de dénoncer nos précarités mais aussi comme un moteur incessant de création et de consommation de textualités sous forme d'images. Mais, même si dans mon imaginaire il y avait quelque chose de très mai 68 entre tant de consignes et jeux de mots dessinés partout, j'ai perçu une technodifférence : la grève avait lieu aussi dans nos écrans, espaces à la fois publics et intimes. Je voulais donc créer une œuvre qui, d'un côté, ait un composant plus documentaire - avec le corpus de photographies numériques prises en manif avec mon Huawei p10 lite - et, d'un autre, soit lié à mon quotidien à travers des traces de mes expériences en tant que femme, professeure, étudiante et migrante sudaca based in Paris dans ces prolongations de nos corps cyborgrisés qu'on appelle GSM, ordinateur, tablette.

C'est après avoir visité l'exposition Le supermarché des images au Musée du Jeu de Paume que j'ai su que la forme que je poursuivais était d'un genre hybride, pas un essai narratif et pas non plus un journal intime, mais entre les deux. Pour mieux situer ma réflexion dans la multiplicité d'images qui façonne notre monde immonde il fallait mélanger le corpus et les genres. Pas seulement dans la disposition pour la lecture, pas 
strictement comme un collage, il s'agissait plutôt de tenter de trans-er les images. J'en ai parlé avec une amie argentine par Whatsapp, photographe beaucoup moins old school que moi, et j'ai tout de suite eu les identifiants de son compte Adobe. Je venais de faire ma première expo solo à Buenos Aires avec une série faite entièrement de Polaroids et je n'avais jamais utilisé Photoshop. C'était aussi important de ne pas cacher dans le projet ses conditions de production symboliques et militantes mais aussi esthétiques.

J'avais un peu trouvé le comment, le avec-quoi, mais il restait encore bien définir le corpus. J'ai fixé une période précise pour les photographies avec lesquelles j'allais travailler : elles ont toutes été prises entre le 5 décembre 2019 et le 8 mars 2020. Je voulais prendre comme point de départ le début de la (g)rève générale et comme fin le début de la grève féministe, mais sans nécessairement les classer d'une manière chronologique pour relativiser l'idée du début et de la fin. J'ai essayé de tracer l'intersection de luttes en ré-instaurant sa synchronie historique qui n'est pas chrononormative. Pour les screenshots c'était (presque) la même période.

Début décembre 2019 j'ai reçu un email avec une enquête obligatoire pour l'obtention de mes diplômes définitifs de Profesora et Licenciada en Letras à l'Université de Buenos Aires en Argentine. Poursuivant un but statistique, l'institution me demandait : étudierez-vous de nouveau votre carrière dans cette université ? En répondant par oui j'ai réaffirmé mon engagement avec l'université en tant qu'espace de partage et de pensée collective. J'ai compris l'impératif urgent de traduire ceci en praxis, c'est-à-dire : ne pas dépeupler l'Université P8 où je suis actuellement en Master Études de Genre. Dans les AG mixtes - enseignant.es et étudiant.es - on venait non seulement de discuter à propos du déroulement des cours face aux luttes mais aussi de voter une validation où les rendus n'était plus obligatoires et les rendus alternatifs étaient désormais possibles. C'était à toustes et à chacun.e de concevoir et suggérer comment procéder : j'avais donc trouvé le cadre pour mon projet. L'atelier de littérature queertpg proposé par C. Flinch dans la salle B034 à Saint-Denis m'imprégnait d'un sentiment qui gagnait la force d'une certitude : produire effectivement cette œuvre comme rendu alternatif était aussi une manière de défendre l'Université comme refuge public où imaginer et tracer de nouvelles formes de penser et où agir autrement devenait possible. Et où je pouvais me permettre de créer à partir des textualités scientifiques mais aussi avec d'autres textualités qui me faisaient juste plaisir. Je voulais alors traduire ce rapport entre désir et université en incluant des échanges avec des ami.es, des citations de fictions lues juste par recommandation - comme Archives des enfants perdus, de Valeria Luiselli, qui m'a fait problématiser mon envie de documenter. Devenait aussi possible d'inclure des recherches qui étaient des échecs : même des résumés de livres pas encore lus - étant soit trop chers soit introuvables en France pouvaient aussi trouver leur place, tout comme dessiner une cartographie de ma rééducation sentimentale en mêlant le ticket d'achat d'une place pour Viril de $\mathrm{V}$. Despentes, le calendrier du séminaire de Paul B. Preciado au Centre Pompidou, l'entrée pour la rencontre avec A. Mbembe, l'exemplaire de Chroniques sur le féminisme noir signé par D. Ribeiro. J'ai même compris que toutes sortes de cyber-communications pouvaient elles aussi gagner de la légitimité pour venir y donner leur témoignage gréviste : les SMS de la SNCF - automatiques et impersonnels mais en même temps adressés -, la convocation de l'OFII pour devenir migrante avec papiers, les emails des profs pour valider nos cours et ceux des étudiant.es pour suivre nos discussions, les événements $\mathrm{FB}$, et même des mèmes. 
6 J'étais attirée aussi par l'affect politique oscillant entre la rage et le désir de revendications queer partout dans les murs: dénoncer les féminicides et toutes sortes de violences patriarcales, la métaphore de la coupe menstruelle débordée, l'actualisation de citations de Simone de Beauvoir, la visibilité TPG, l'usage du langage inclusif dans les graffitis. À l'Université, on s'est interrogé.es sur les technologies artistiques et politiques possibles à performer pour s'exprimer mais aussi pour mieux interpeller. Dans ce sens j'ai vu confirmé que la férocité concrète des images ne devait pas se mélanger avec de longues citations ni être accompagnée de références concrètes pour laisser plus de place aux visualiseur.trices pour les situer et les signifier.

7 Alors que le projet est presque entièrement en français, il y a quelque chose de très global/transnational dans les revendications queer/cuir. C'est pourquoi je voulais que le projet soit aussi capable de visibiliser la géographie poreuse des subjectivités migrantes qui sont façonnées et traversées en même temps par plusieurs lois/discours/ territoires. Cette envie s'est traduite non seulement en incluant plusieurs langues, mais aussi à partir de l'exploration des réseaux sociaux comme locus-délocalisés où les liens d'affectivité peuvent aussi avoir lieu. Peu importe qu'on soit à Paris ou à Buenos Aires, c'est, comme disait N. Perlongher: «No queremos que nos persigan, ni que nos aprendan, ni que nos discriminen, ni que nos maten, ni que nos curen, ni que nos analicen, ni que nos expliquen, ni que nos toleren, ni que nos comprendan: lo que queremos es que nos deseen ${ }^{1}$.»

\section{NOTES}

1. « Nous ne voulons pas être chassé.es, ni appris.es, discriminé.es, tué.es, soignées, analysé.es, expliqué.es, toleré.es, pas plus que compris.es : ce que nous voulons est d'être désiré.es. » [ma traduction]

\section{INDEX}

Thèmes : Actualités

Palabras claves : huelga, precariedad, universidad, género, Francia

Mots-clés : grève, précarité, université, genre, France

Keywords : strike, precariousness, university, gender, France 


\section{AUTEUR}

\section{AGUSTINA MATTAINI}

UBA/PARIS 8/LILLE 3

mattainiagustina@gmail.com

Féministe, migrante et photographe diplômée en Lettres par l'Universidad de Buenos Aires

(Argentine), elle estenseignante à l'Université de Lille et masterante en Études de genre à

l'Université Paris VIII. Ses recherches gravitent autour de la représentation de la précarité dans

la littérature argentine contemporaine. 